\title{
Giant condyloma acuminatum of vulva
}

\section{S. M. Ramiz Ahmed, Mohammad Saiful Islam, S. M. Yunus Ali, Nargis Akhtar and Md. Sajid Hasan}

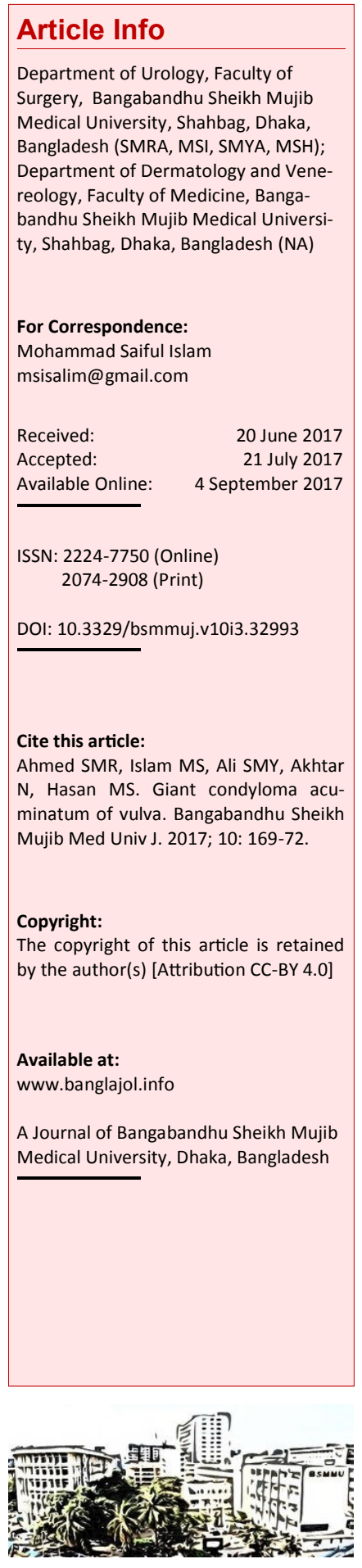

\section{Abstract}

In this paper, A 23 year old married woman who was diagnosed as a case of giant condyloma acuminatum of vulva measuring about $15 \times 8 \times 3 \mathrm{~cm}$, irregular surface with multiple projections, oval in shape, firm to hard in consistency, mildly tender, exophytic, cauliflower like growth involving the whole vulva (lower part of mons pubis, labia, vestibule, clitoris, around vaginal opening). Another multiple small lesions were present at perineal region but there was no inguinal lymphadenopathy. She underwent a combined electro cauterization and cryotherapy for small to moderate size multiple primary and recurrent warty lesions and wide surgical excision with fasciocutaneous advancement flaps procedure for a giant lesions in the vulva. Excisional biopsies were performed to detect potential malignancy but malignancy was not found histologically. The patient was advised to first follow-up 1 month after operation when multiple small warty lesions were developed and treated and the subsequent follow-up for 3 months.

\section{Introduction}

Condyloma acuminatum (genital wart or or veneral wart) is a benign superficial skin manifestation accredited to the epidermotropic human papilloma virus (HPV) which is a double-stranded DNA virus belonging to the Papillomaviridae family. Infection of HPV begins at the basal cell layer of stratified squamous epithelial cells and stimulates cell proliferation in the epithelium. More than 40 types of HPV can infect the genital area and be sexually transmitted. Types 6 and 11 are not oncogenic and contribute to about $90 \%$ of anogenital warts. 1

In the general population, the prevalence of HPV infection among asymptomatic women ranges from 2 to $44 \%$ and among men from 2.3 to $34.8 \%$. Although there is no exact data but it is rare in our country. Moreover, such a giant wart of this patient was first noticed in our country which was very much difficult to treat surgically but was performed successfully with advancement flap without any gross disfiguration and post-operative complication. About $70 \%$ of HPV infections rectify spontaneously in 1 year and $90 \%$ in 2 years and HPV persistence develops in the remaining persons.? In the follow-up period, the patient was manifested with multiple recurrences in the new and previously operated sites.

HPV infection can affect both sexes. The proportion of male to female is about 2.3: 1 . Peak incidence age is 20-24 years. Transmission of infection from one person to another through direct contact of sex including genital organs, anal, or oral sex exercise with a person who is infected. .4 In this case, the woman was infected from his husband penile wart during sexual intercourse.

Although patient was not immunized with HPV vaccine due to her poor economic condition after repeated recurrence but it is still now a great challenge to free anyone from HPV infection without HPV vaccine.

\section{Case Report}

A 23 year old married women presented with a 3 months history of itching in some part of the vulva early on 2014 which initially was rubbed by herself to relieve the itching that causes redness of the part of the vulval skin with vesicular eruptions (Figure 1). Fluid discharge from rupture of this vesicular eruption during and after itching spread to other part of the vulva causing generalized vulval itching and redness. Vesicular eruptions gradually turn into multiple soft, small, projectile tag like structures out of which $2-3$ grew as $0.3-0.5 \mathrm{~cm}$ in length and treated by surgical excision with diathermy. Due to rapid growing and multiple in number, she visited to the outpatient Department of Dermatology and Venereology, Bangabandhu Sheikh Mujib Medical University, where 1st cycle $\mathrm{NO}_{2}$ cryotherapy was given. But whole area of the vulva gradually became thickened, hardened, changes in color 
and texture and painful for 4-5 days after cryotherpy. She was, then, referred to outpatient Department of Urology of the same University for better treatment.

She has no family history of such type of disease except her husband and has two children in 9 years of her married life. Her husband and she develop those lesions simultaneously. In her husband the lesions were at the root of penis which was treated by $\mathrm{NO}_{2}$ cryotherapy at the Department of Dermatology and Venereology at the same time of her treatment. She is normally menstruating women, immunized against all EPI disease and has no history of hormonal contraceptives, multiple sexual partners, early coital age but occasional anal intercourse.

On physical examination, there is an enormous, exophytic, cauliflower like growth invoving the whole vulva (lower part of mons pubis, labia, vestibule, clitoris, around vaginal opening) measuring about $15 \times 8 \times 3 \mathrm{~cm}$ in diameter with irregular

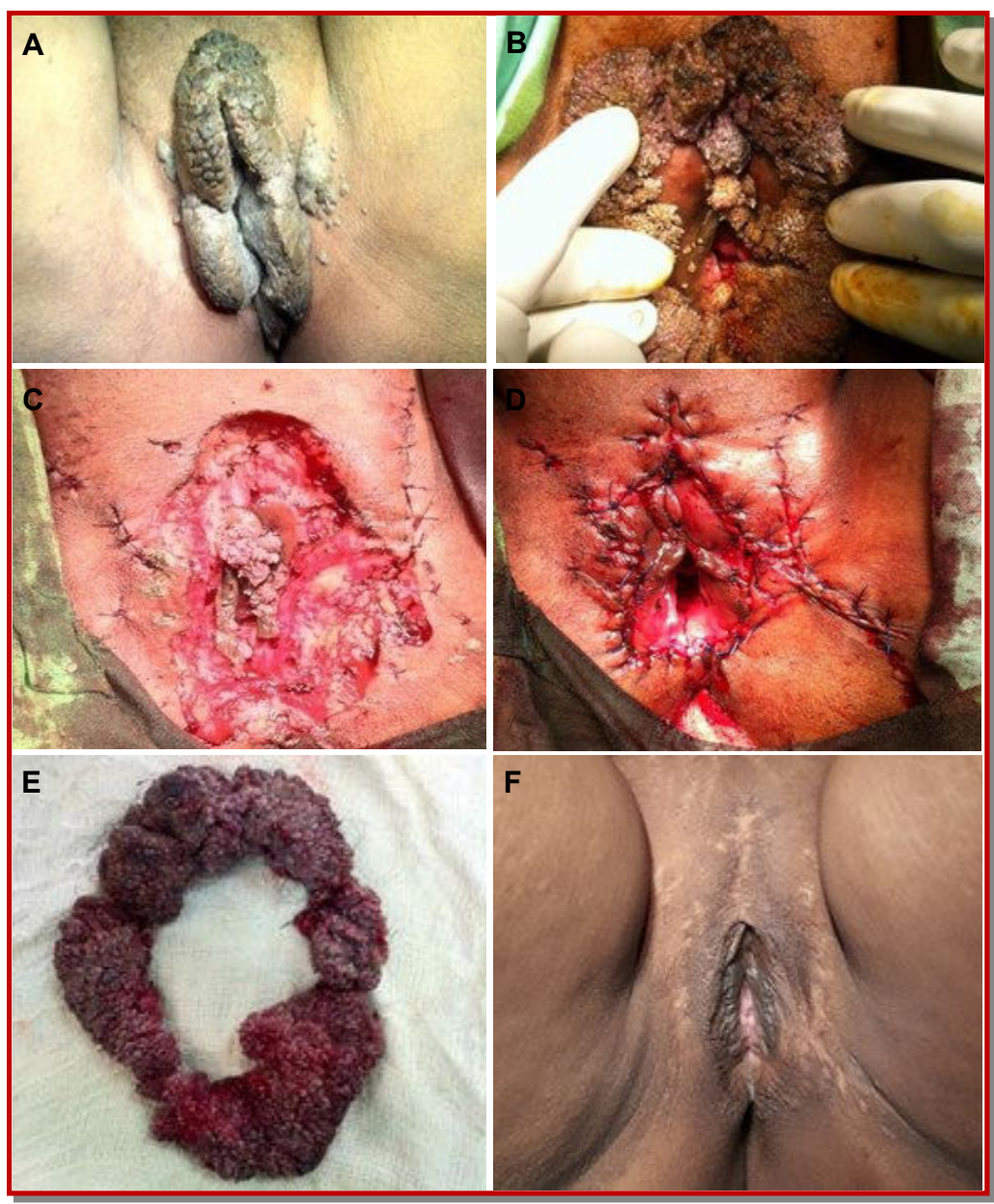

Figure 1: Giant condyloma acuminatum of vulva (Pre-operative; A, B). Total surgical excision and wound closure with fasciocutenous advancement flap (C, D). Excised part of the condyloma (E) and appearance of vulva after operation (after 3 months, F) surface with multiple projections, oval in shape, firm to hard in consistency, mildly tender (Figure 1). Another multiple small lesions are present at perineal region. There is no inguinal lymphadenopathy.

All laboratory examinations of blood and urine analysis were normal. Serological test of syphilis (TPHA), hepatitis (HBsAg, Anti HCV Ab), chlamydia trachomatis (chlamydial $\mathrm{Ab}$ ), acquired immunodeficiency syndrome (HIV 1 and 2) were negative.

\section{Operative technique}

Proper pre-operative evaluation and counseling of the patient were done. After informed consent, spinal anesthesia was given. Keeping patient in the lithotomy position, the surgical painting, draping and catheterization were done. Then complete surgical excision of the large wart was done first with diathermy and multiple small warts thereafter. Two fasciocutaneous advancement flaps were made on lateral sides of vulva. Proper hemostasis was ensured. Wound was closed with advancement flaps by 4-0 interrupted polypropylene suture. Wound was cover with sterile dressing. Tissue was sent for histopathological examination with proper tissue preservative and labeling.

In the post-operative period, the patient was covered with intravenous ceftriaxone and amikacin for 5 days followed by 7 days of oral cefixim. The patient was nothing by mouth for 4-5 hours followed by oral soft diet and advised to early ambulation. First check dressing was done on $5^{\text {th }}$ post-operative day and subsequent dressing was done up to $12^{\text {th }}$ post-operative period to assess the wound healing and complication. On $13^{\text {th }}$ post-operative day, all stitches were removed and advised to apply povidone iodine ointment to wound sites twice daily. The post-operative course was uneventful and the cosmetic result was satisfactory with no complication. She was discharged on $16^{\text {th }}$ post-operative day and advised to follow-up after 1 month.

Histopathological examination of the tissue from growth around vulva showed polypoid piece of tissue lined by stratified squamous epithelium which had dysplastic changes and koilocytosis. The stromal core was fibrovascular and infiltrated with chronic inflammatory cell. No malignant cell was seen. The histopathology was typical for a condyloma accuminatum.

\section{Follow-up}

On first follow-up (1 month after discharge), after examining the genital and perineal area, surgical wound was well healed but multiple small warts were found scattered in the vulval region, perineal region and around anus. With these clinical features she was again referred to the skin and veneral disease department for cryotherapy where 20 cycle 
$\mathrm{NO}_{2}$ cryotherapy was given in 2014 to 2015 at 2 weeks interval. On subsequent 3 monthly followup 2015-2016, multiple relatively larger size warts were developed on the similar area during course of cryotherapy in each follow-up and treated surgically by diathermy cauterization (Figure 2). In one follow-up, a small size wart was appeared on the left nipple and another on the back of the chest (new site) and treated surgically by diathermy coagulation 2016 and early 2017 respectively. The patient was then advised to follow-up 3 monthly. Patient was also advised to give HPV quadrivalent (HPV4) 3 dose vaccines after HPV genotyping to prevent recurrence but patient was unable to bear that cost.

\section{Discussion}

Such a giant condyloma acuminatum with benign appearance which is a rare entity with an average incidence of $0.1 \%$ in the general population, steadily the number of cases increases in the last few decades but the prevalence of HPV infection is unknown in our country. The immune system effectively repels the majority of HPV infections by localized cell-mediated immune responses. About $10 \%$ of individuals may develop a persistent infection with a risk of proliferative lesions, highgrade precursors and eventually an invasive carcinoma. So, a large representative biopsy specimen is crucial to justify the structure of the growth to establish the diagnosis and to exclude foci of verrucous carcinoma. In our patient, histological examination of biopsy tissue reveals no malignancy.

HPV infection usually observed in patients with underlying immune deficiency status that are either acquired (such as AIDS or immune suppression associated with organ transplantation) or congenital immunodeficiency. In this reported case, the patient was healthy and had no clinical or laboratory features suggestive of immunodefiency. Giant condyloma invades by expansion rather than by infiltration, leaving basement membrane intact, and shows a well-stratified epithelium with

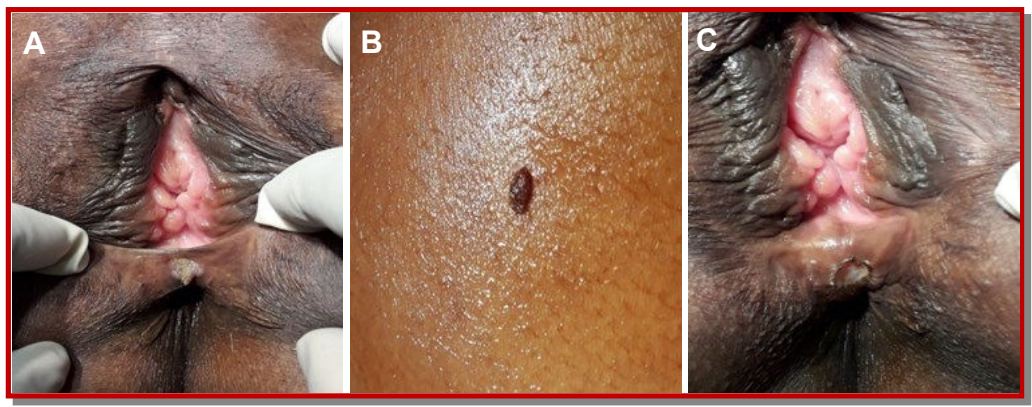

Figure 2: Recurrent condyloma wart at perineal region (A) and back side of right chest (B); Electro-cauterization of recurrent perineal wart (C) minimal cellular dysplasia or atypical cells. In our reported case, it was not possible to determine the subtype of the HPV virus (HPV genotyping).

It has the known propensity for local invasion and recurrences $(30-70 \%)$ combined with complications resulting from non surgical therapy and surgery which indicate the need to define optimal treatment for these lesions. There is still no general agreement on the best method of treatment, as the disease is relatively rare. The study by $\mathrm{Chu}$ and colleagues showed that approximately 50 percent of all patients condyloma acuminatum of vulva treated with radical surgery as the initial mode of treatment developed recurrences. $\frac{5}{-}$

Condyloma acuminatum of vulva has been treated by a variety of modalities but the effect of medical therapy (podofilox $0.5 \%$ solution or gel, imiquimod cream $5 \%$ ), local liquid $\mathrm{N}_{2}$ cryotherapy, $\mathrm{CO}_{2}$ laser therapy and surgical treatment is uncertain. A study on 42 published cases of condyloma accuminata of vulva concluded that the only consistently effective therapy is wide surgical excision of tumor with clear margins with or without adjuvant chemotherapy .6

Wide surgical excision with closure of wound defect by fasciocutaneous advancement flaps from both lateral sides of vulva was done comfortably. Mestrovic et al. (2003) reported six cases of giant condyloma accuminatum and evaluated the use of mesh-skin grafts in covering the skin defect after radical local excision of perianal giant condyloma acuminatum comparing to other methods of treating the skin defect. In mesh-skin graft, there were no recurrences, wound infections, wound bleeding, hypertrophic scars, or mesh-like skin appearance of the recipient site. Therefore a good cosmetic and functional result was achieved. $\underline{6}$

Cryotherapy, electro-cauterization and wide surgical excision methods were used only in this patient for recurrent lesion. In a case report of 24 years young lady with generalized verruca vulgaris in Bangladesh by Paul et al. (2017), showed that even after use of cryotherapy, electrocautery, electrofulguration, electrodessication etc., the lesion did not cure.?

Patient was also advised to give HPV quadrivalent (HPV4) 3 dose vaccines after HPV genotyping to prevent recurrence but patient was unable to bear that cost. The patient attends the outpatient Department of Urology, 3 monthly for regular follow-up. In a case report of 24 years young lady with generalized verruca vulgaris in Bangladesh by Paul et al. (2017) showed that HPV-9 valent vaccine (4 doses at 0, 2, 6 and 12 months) had 50\% improvement.?

Vaccines are available against HPV (for both males and females between the ages 9 and 26 years) to 
help prevent cervical cancer in female and genital warts in both sexes multiple types of vaccines are available for HPV infection prevention. In 2006, the FDA approved a quadrivalent HPV vaccine that prevents HPV types 6,11,16, and 18 infections and in 2014, a nonavalent HPV vaccine that prevents HPV types 6, 11, 16, 18, 31, 33, 45, 52, and 58 infections. $\frac{8,9}{}$ In a article by Lee et al. (2011) reported on a 46 year old man with extensive perianal condylomata acuminatum that showed a dramatic improvement after a single injection of the recombinant quadrivalent human papillomavirus (HPV) vaccine (against the HPV types 6, 11, 16, and 18). In contrast, their experiences with the prophylactic HPV vaccine for treating condylomata acuminatum are less promising. $\underline{10}$

As the incubation period of HPV varies between 3 weeks and 8 months and the majority of anogenital warts appear 2-3 months after exposure. In the follow-up period, multiple small lesions were appeared in the remaining vulval and perineal areas within 1 month after operation which were treated combindly by cryotherapy and electro cauterization. Although the patient's husband had a small warty lesion at the root of the peins and was treated effectively by cryotherapy and there was no recurrent. So, the patient was also advised to use condom during sexual intercourse to prevent sexual transmission from her huasband, maintainance of personal hygiene to prevent transmission indirectly through contaminated object and surfaces and avoiding use of contraceptive pills.

\section{Conclusion}

Surgical excision is the treatment of choice but other treatment modalities such as cryotherapy and laser have yielded mixed results with variable success and recurrence rates. The lesion has a tendency to recur but is not frequently associated with metastatic potential. Post-treatment clinical monitoring (follow-up) is highly recommended.

\section{Ethical Issue}

Written and signed informed consent from the patient was taken for publishing this case report.

\section{References}

1. Leonard B, Kridelka, Delbecque K, Goffin F, Demoulin S, Doyen J. A clinical and pathological overview of vulvar condyloma acuminatum, intraepithelial neoplasia and squamous cell carcinoma. Bio Med Res Int. 2014; 2014.

2. Wein AJ, Kavoussi LR, Patrin AW, Peters CA. Campbell-Walsh Urology. 11th ed. Philadelphia, Elsevier Inc., 2016, pp 378-80.

3. Sinal SH, Woods CR. Human papillomavirus infections of the genital and respiratory tracts in young children. Semin Pediatr Infect Dis. 2005; 16: 306-16.

4. Haque W, Kelly E, Dhingra S, Carpenter LS. Successful treatment of recurrent Buschke-Lowenstein tumor by radiation therapy and chemotherapy. Int J Colorectal Dis. 2010; 25: 539-40.

5. Chu QD, Vezeridis MP, Libbey NP, Wanebo HJ Giant condyloma acuminatum (Buschke-Lowenstein tumor) of the anorectal and perianal regions: Analysis of 42 cases. Dis Colon Rectum. 1994; 3: 950 -57 .

6. Mestrovic T, Cavcic J, Martinac P, Turcic J, Reconstruction of skin defects after radical excision of anorectal giant condyloma acuminatum: 6 cases. J Eup Acad Dermatol Venerol. 2003; 17: 541-54.

7. Paul HK, Akhtar N, Zakaria ASM, Biswas SK. Generalized verrucus vulgaris: A case report. Dhaka Community Med Coll J. 2017; 6: 29-32.

8. Lacey C, Woodhall S, Wikstrom A, Ross J. European guideline for the management of anogenital warts. J Eur Acad Dermatol Venereol. 2012; 27: 263 70.

9. Centers for Disease Control and Prevention, Workowski KA, Berman SM. HPV infection and genital warts. Sexually transmitted diseases treatment guidelines 2006. MMWR Morb Mortal Wkly Rep. 2006; 55(RR-11): 62.

10. Lee HJ, Kim JK, Kim DH, Yoon MS. Condyloma accuminatum treated with recombinant quadricvalent human papillomavirus vaccine (types 6, 11, 16, and 18). J Am Acad Dermatol. 2011; 64: 130-32. 